Fecha de recepción: mayo 2021 Fecha de aceptación: junio 2021 Versión final: julio 2021

\section{Adaptación y Aprendizaje del Diseñador a partir de la Nueva Realidad}

Alejandrina Bautista Jacobo ${ }^{(1)}$, Arodi Morales Holguín $^{(2)}$, Manuel Alejandro Vázquez Bautista ${ }^{(3)}$

Resumen: El objetivo de este trabajo es conocer cómo el estudiante de Diseño se adaptó a las clases virtuales y cuál fue su percepción de su aprendizaje a partir de la nueva realidad producto del COVID-19. Más de la mitad de los estudiantes consideran que desde una perspectiva integral, las clases en línea no permiten alcanzar un aprendizaje como en el espacio presencial, donde se identifica la heterogeneidad de las asignaturas y enseñanza propia del área de diseño. Se sugiere implementar métodos innovadores que permitan mejorar el aprendizaje y la experiencia tanto de los estudiantes como de los docentes en el actual método educativo virtual.

Palabras clave: diseño - aprendizaje - clases virtuales - educación superior - universitarios - COVID-19 - pandemia.

[Resúmenes en inglés y portugués en la página 175]

(1) Licenciada en Matemáticas, Universidad de Sonora. Maestría en Matemática Educativa, Universidad de Sonora. Posgrado en Educación Instituto Pedagógico de Posgrado de Sonora, A. C. Profesora Investigadora de Tiempo Completo de la Universidad de Sonora.

(2) Doctor en Arquitectura, Diseño y Urbanismo por la Universidad Autónoma del Estado de Morelos. Maestro en Administración, Master en Publicidad y Marketing y Licenciado en Diseño Gráfico. Es Profesor Investigador de Tiempo Completo de la Universidad de Sonora. Es distinguido como miembro del Sistema Nacional de Investigadores de México.

(3) Ingeniero Industrial y de Sistemas Universidad de Sonora. Maestro en Ciencias Centro de Investigación en Alimentación y Desarrollo A. C. Maestros de la Universidad Vizcaya de las Américas. 


\section{Introducción}

En diciembre de 2019, un brote de enfermedad debido a una nueva cepa de coronavirus surgió en Wuhan, China. De acuerdo con la Organización de las Naciones Unidas (2020), la actual pandemia por COVID-19 ha tenido un notable efecto adverso en la educación, ya que el cierre de todas las instituciones educativas forzó a una repentina transición de clases presenciales a clases virtuales. Esta situación ha afectado en mayor medida a países con bajos recursos, ya que existe una menor accesibilidad a conexión de internet, dispositivos electrónicos y, en ciertos casos, espacios en el hogar. Esta situación provoca aún enormes desafíos tratándose de adaptar a esta nueva modalidad de enseñanza-aprendizaje y esto se debe a que muchos de los docentes y estudiantes no contaban con los medios tecnológicos, ni con la preparación, en el caso de los docentes, de impartir una clase en línea. En cuanto a los estudiantes, estos enfrentan muchos problemas, como la falta de motivación y de entornos de aprendizaje adecuados, ya que están aislados (Bao, 2020). Por otra parte, México, por ser un país en vías de desarrollo, una gran parte de la población estudiantil se ha enfrentado a los problemas de accesibilidad anteriormente mencionados (Zamora, 2020). Otra situación preocupante que se ha reportado en los estudiantes es la alta prevalencia de ansiedad y estrés debido a las clases virtuales a consecuencia de la pandemia. En un estudio realizado en la Universidad Autónoma de Chiapas, se encontró que uno de los comportamientos observables en los estudiantes debido al uso de las plataformas virtuales es el estrés, situación de tensión física y emocional vinculada a las exigencias del mundo académico (González, 2020). Un detonante para esto ha sido principalmente los exámenes o la entrega de trabajos finales (Alba-Linero, Moral-Sánchez \& Gutiérrez-Castillo, 2020; Unger \& Meiran, 2020).

En cuanto a la percepción del estudiante con respecto a su aprendizaje obtenido en las clases virtuales, éste ha sido en su mayoría negativo. La percepción del estudiante es que no está recibiendo un aprendizaje de calidad (Abreu, 2020) o que las clases en línea no son tan efectivas como las clases presenciales. A ello hay que agregar los problemas del profesorado en la enseñanza dentro de la nueva modalidad; en el caso de México, el estudio de Morales y González (2021) identifican dos perfiles de profesores: los pre-digitales y digitales. El objetivo de este trabajo es explorar la adaptación que tuvo el estudiante del área de diseño y su percepción de aprendizaje durante las clases en modalidad virtual.

\section{Método}

Se llevó a cabo un diseño metodológico cuantitativo de tipo exploratorio en una muestra no aleatoria de 322 estudiantes de diseño de diferentes semestres de la Universidad de Sonora, México. El 39.4\% ( $\mathrm{n}=127)$ eran del sexo femenino y $60.6 \%(\mathrm{n}=195)$ masculino, todos en un rango de edad de 18 a 31 años $(\mathrm{M}=20.45 ; \mathrm{DE}=1.40)$. La mayoría (70.5\%) manifestó dedicarse únicamente al estudio. 
Este estudio tiene como objetivo analizar la adaptación de los estudiantes de diseño a las clases virtuales debido a la pandemia y cómo percibe su aprendizaje en esta nueva modalidad. Para ello, se diseñó una encuesta con preguntas abiertas y cerradas para conocer sobre esta temática. Además, se agregaron al cuestionario algunas preguntas sociodemográficas como sexo, edad y si estaba trabajando.

Dada la situación por la pandemia, la aplicación del cuestionario fue efectuado en la plataforma de Google forms. Para hacer llegar el cuestionario, se utilizó el correo electrónico en donde se explicó los objetivos de la investigación y se hizo la aclaración que éste era de manera voluntaria y anónima. El procesamiento de datos se llevó a cabo por medio del paquete estadístico SPSS V.25. Se reportan frecuencias y porcentajes para las variables categóricas.

\section{Resultados}

\section{Adaptación de los estudiantes de Diseño a las clases virtuales}

La primera pegunta que se le hizo al encuestado fue: ¿Qué plataforma utilizaste para tus clases virtuales? Mencionaron que algunos profesores combinaban las plataformas de acuerdo a sus necesidades o capacidad de manejo, por ejemplo, si el profesor no conocía la herramienta de una plataforma para elaborar examen o enviar tareas, utilizaba otra con la que ya tenía más experiencia. Las plataformas más utilizadas en combinación con otras fueron: Zoom (77\%), Teams (40.68\%) y Classroom (38.88\%). Otras plataformas utilizadas fueron: AVAUS, SIVEA, Moodle, Edmodo, Google Meet, EMINUS, Drive y Schoology. El 76.4\% de los estudiantes utilizó una laptop para sus clases virtuales, un $12.4 \%$ mencionó la computadora tipo desktop y hubo un porcentaje del 11.2\% que refirió haber tenido la necesidad de emplear su celular. Esto último se debía en la mayoría de las ocasiones (55.6\%) por la falta de Internet en ese momento, sin embargo, había otros que referían el hecho de que su laptop la tenía algún familiar (27.8\%) y hubo pocos que mencionaron que se encontraban trabajando a la hora de la clase o porque en estaban fuera de su casa y por consecuencia se conectaban a la clase por medio del celular (16.7\%).

Sólo un $12.7 \%$ de los estudiantes de Diseño se vieron en la necesidad de trasladarse a otro lugar para continuar con sus clases virtuales, esto debido a la falta de Internet en su localidad. Los lugares a donde acudieron para continuar con sus clases fueron principalmente a casa de un familiar (73.2\%), en segundo orden la casa de un amigo(a) (19.5\%) y sólo el $7.3 \%$ se fue a un Cibercafé.

A pesar que el estudiante de dicha area posee habilidades en las Tecnologías de Información y Comunicación (TICs), dado el perfil de formación y el necesario manejo de diversas plataformas y softwares de gran complejidad como la compuesta por la gama Adobe y otras, un $24.5 \%$ de los estudiantes manifestó que el proceso de adaptación a las clases virtuales le fue muy difícil o difícil, un $37.6 \%$ se mantuvo indeciso a esta pregunta 
y un porcentaje ligeramente mayor (37.9\%) mencionó que su proceso de adaptación fue fácil o muy fácil.

Una de las razones de lo anterior podría estribar en que la formación del diseñador desde sus orígenes en la Bauhaus hasta antes de la pandemia se ha centrado en el sistema de taller (Tovey, 2015), dando así forma a una dinámica proyectual que incentiva la creatividad desde un ambiente libre y de cierta manera independiente, donde interactúan alumnos y profesor desarrollando proyectos en una dinámica de retroalimentación verbal y visual muy proactiva. Ambiente que ha sido relegado por espacios virtuales que imposibilitan apreciar y evaluar aspectos subjetivos que parece sólo ser posible en el entorno presencial. Dicha transición del taller hacia lo virtual para muchos diseñadores resulta ser difícil e incluso estresante, pues se asume se diluye el ambiente propio del taller.

Por otro lado, la enseñanza del diseño en lo referente a contenidos de corte tecnológico y manejo de software, los cuales componen una importante porción de la malla curricular, resulta ser altamente personalizada; más aún cuando los softwares que utilizan los diseñadores son de características heterogéneas y de una alta complejidad. En esta coyuntura, la transición a entornos virtuales y que disuelve en buena medida dicha asesoría personal, igualmente pudiera generar desinterés e incluso estrés en el estudiantado. No obstante, como se puede apreciar en los datos presentados, otro sector de alumnos se ha adaptado sin muchas dificultades a la nueva realidad, por lo que se asume que el acceso a un buen equipo de cómputo y buena conexión de internet podría actuar como otra variable en estos resultados, siendo entonces el factor económico relevante para la capacidad de adaptación y evaluación de la formación a través de entornos virtuales.

Estudios similares han reportado porcentaje de adaptación más elevados, como es el reportado en estudiantes Albanos, en donde más del 52.5\% refirió que el proceso de adaptación del aprendizaje en línea le fue muy fácil (Xhelilli, Ibrahimi, Rruci \& Sheme, 2021). En el caso de los diseñadores quienes cursan asignaturas que se desenvuelven en entornos muy diversos, la adaptación es más compleja frente a estudiantes de otras disciplinas quienes llevan buena parte o el total de las asignaturas de forma tradicional.

\section{Niveles de ansiedad o estrés en los estudiantes de Diseño debido a las clases virtuales a causa de la pandemia}

El 78\% de los estudiantes de Diseño refirió presentar algún nivel de ansiedad o estrés debido a las clases virtuales. La evaluación del estado psicologico en población estudiantil ha sido estudiada desde antes de la pandemia. Algunos de los factores que se han identificado como generadores son los problemas de adaptación, la preocupación por el futuro, los exámenes, entre otros (Uskun, Kisioglu \& Ozturk, 2008). Todas estas situaciones que ya estaban presentes durante un curso normal se vieron agravadas por el repentino cambio a una enseñanza virtual. 
Estudios recientes reportados sobre población estudiantil han informado un aumento de la ansiedad durante la pandemia en jóvenes universitarios y con mayor frecuencia en menores de 35 años (Huang \& Zhao, 2020; Unger \& Meiran, 2020). En este estudio, el proceso de adaptación a las clases virtuales se asoció a la presencia de este estado psicológico $(\mathrm{p}<0.05)$. El 96\% de los estudiantes cuyo proceso de adaptación a las clases virtuales fue difícil o muy difícil manifestaron presentar ansiedad o estrés mientas que a los que le fue fácil este porcentaje fue del 62.3\% (Tabla 1). Por otra parte, estos estados emocionales, tales como el sentimiento de tristeza, ansiedad, frustración, cansancio y/o falta de motivación, se han reflejado en la falta de atención y de interés en las clases impartidas virtualmente, lo que ha comprometido el seguimiento efectivo de su formación académica (Valle, 2020).

\begin{tabular}{|l|cc|c|}
\hline \multirow{2}{*}{ Proceso de adaptación a las clases virtuales } & \multicolumn{2}{|c|}{ Presenta ansiedad o estrés } & \\
& $\mathrm{Si}$ & $\mathrm{No}$ & Total \\
\hline Difícil o muy dificil & $76(96.2 \%)$ & $3(3.8 \%)$ & 79 \\
Indeciso & $99(81.8 \%)$ & $22(18.2 \%)$ & 121 \\
\hline Fácil o muy fácil & $76(62.3 \%)$ & $46(37.7 \%)$ & 122 \\
Total & $251(78 \%)$ & $71(22 \%)$ & 322 \\
\hline
\end{tabular}

Tabla 1. Prevalencia de ansiedad o estrés según el proceso de adaptación a las clases virtuales en una muestra de 322 estudiantes de Diseño. Fuente: elaboración propia, los porcentaje están calculados con respecto al renglón

\section{Qué tan de acuerdo están los estudiantes de diseño en las clases virtuales}

Los estudiantes expresaron su opinión acerca de la modalidad en línea que se puso en práctica en marzo del 2020 consecuencia de la pandemia por Covid-19. Las opiniones no resultan favorables para el método de enseñanza actual, ya que casi un $41 \%$ de los estudiantes del área de Diseño no estaban de acuerdo con las clases virtuales y un $32 \%$ se reportó de indecisos debido a que existen diferentes situaciones que obstaculizan un aprendizaje íntegro. Entre los comentarios sobre los principales motivos por los cuales no estaban de acuerdo, se destacan los problemas técnicos, como la falta de luz, la conexión inestable o fallas en las plataformas. Hallazgos similares fueron reportados por Xhelilli, Ibrahimi, Rruci \& Sheme (2021). Algunos estudiantes referían que si bien es cierto tenían un manejo aceptable en las plataformas, las fallas repentinas del Internet les preocupaba, sobre todo en los días en donde tenía que reportar un examen. 
Dada la inmediatez con la que se manejó la situación, no existió preparación alguna para trasladar las clases en su totalidad a una modalidad virtual, en consecuencia, la adaptación del alumnado y de los docentes aún se mantiene en espera. Aunado a esto, los alumnos manifiestan una escasa concentración, debido a que en casa existen diversos distractores y algunos no cuentan con un lugar fijo para tomar las clases correspondientes. Según los estudiantes, la virtualidad rompió con los horarios y llevó al límite la carga académica propuesta por los profesores, en consecuencia, las habilidades duras y blandas no tienen un desarrollo óptimo. La interacción alumno-docente y alumno-alumno se ha visto afectada, el acercamiento o atención personal del docente es prácticamente nulo, y el aprendizaje es poco eficiente, mencionan los estudiantes.

\section{Aprendizaje percibido por los estudiantes en la modalidad virtual}

Otra pregunta que se realizó a los estudiantes fue ¿consideras que las clases virtuales te permiten tener un mayor aprendizaje en las materias?, a lo cual, sólo el 1.6\% mencionó que sí, un $55.6 \%$ considera que las clases virtuales no permiten un aprendizaje y hubo un $42.5 \%$ que refirió que depende de las materias. En este hallazgo puede identificarse que el perfil de la asignatura es un factor importante, pues aquellas de perfil teórico, tales como historia del arte, semiótica, métodos, entre otras, es posible alcanzar un mayor aprendizaje dado que el sistema de enseñanza empleado en el aula presencial en estas asignaturas, donde el profesor proyecta imágenes apoyándose en recursos tecnológicos y en explicaciones ante los alumnos, tiene estrecha similitud con el sistema que se sigue en línea, donde la pantalla del monitor cumple las funciones de la proyección en el aula, y la exposición del profesor presencial como virtual se desenvuelve de forma análoga. En cambio, las asignaturas de taller, que tiene como objetivo desarrollar proyectos prácticos simulando a aquellos que se desarrollan en el ejercicio profesional y donde el profesor hace las veces de guía académico, asesor de proyecto personal y motivador, dicho conjunto se diluye al transitar del aula a las plataformas virtuales.

Algo similar ocurre con los contenidos relacionados con el aprendizaje de software, donde la asesoría personal y la práctica es preponderante para un verdadero aprovechamiento, y que se debilita al conducirse mediante el aula virtual, pues el profesor tiene sólo un espacio (la pantalla de computadora) para enseñar al grupo y la asesoría personalizada. Por otro lado, al quedar expuestos los alumnos ante las miradas de los demás, algunos omiten hacer peguntas al profesor, lo que degrada aún más el aprendizaje. De este modo no resulta extraño que más de la mitad de los entrevistados consideren que desde una perspectiva integral las clases virtuales no permiten alcanzar un aprendizaje como en el espacio presencial.

En esta investigación, el 86.5\% de los estudiantes de Diseño considera que no tuvo el aprovechamiento que hubiera tenido en clases presenciales, consideran que en esta modalidad las clases no se logran comprender. Algunas frases textuales de los estudiantes fueron: 
"Todas mis clases deben de ser prácticas, no es el mismo rendimiento, se presta menos atención..."; "Simplemente mi carrera no puede llevarse en línea, necesito mis prácticas, hay muchas cosas visuales que no pueden utilizarse en una plataforma, en ocasiones se necesita atención especializada y simplemente no puedo llevar materias donde solo me piden entrar a dejar una exposición" y "Se dificulta entender las clases, si falla el Internet pierdo parte de la clase, los talleres son más complicados de llevar en línea, resolver nuestras dudas es más difícil, no tengo lo necesario en mi casa para algunos de mis trabajos".

Ahora bien, no todos los comentarios fueron negativo, algunos comentarios a favor de las clases virtuales fueron principalmente por la protección para ellos y su familia, además de aprender a usar las plataformas virtuales. Otros hacían referencias a que estudiar desde casa es más cómodo y se ahorran costos como el del transporte.

\section{Conclusiones}

En este trabajo nos permitió conocer cómo se adaptaron a las clases virtuales los estudiantes de diseño, situación influenciada por la pandemia resultado del COVID-19, además de conocer su percepción sobre el aprendizaje en esta nueva modalidad. Los estudiantes vivieron un reto para adaptarse que les provocó un estado psicológico de ansiedad y estrés. Muchos estudiantes regresaron a sus hogares para continuar con las clases en línea y el detalle fue que en sus casas no tenía el recurso tecnológico ni el acceso a Internet. Además, algunas familias solo contaban con una laptop y tenían que compartirla lo cual se hacía difícil en situaciones donde se ocupaban la misma hora. Otra situación es que algunos estudiantes se encontraban trabajando al momento de la clase, por lo que prestaban poca atención a esta, la cuales eran atendidas a través del celular.

Al hablar sobre la percepción del aprendizaje en la modalidad virtual, se identificó que los diseñadores, a pesar de ser estudiantes que manejan una amplia gama de softwares de alta complejidad, más de la mitad considero difícil este sistema de enseñanza, señalando que las clases virtuales no permiten un verdadero aprendizaje, siendo considerado de manera positiva apenas por menos del $2 \%$. En consecuencia, la mayoría tiene una percepción de que su aprendizaje fue muy pobre y que éste hubiera sido mejor en clases presenciales. Sienten que de forma presencial hay más interacción entre el profesor y sobre todo entre los compañeros por lo que se resuelven dudas más rápido, además, existen materias que se requiere de espacio amplio lo cual mucho no cuentas con eso en sus hogares.

Se trata de una adaptación que, para los diseñadores, dada la diversidad de la enseñanza que se sigue en su programa de estudio, ha resultado ser más compleja que aquellas disciplinas apoyadas en el formato tradicional. Por todo esto, es de vital importancia encontrar e implementar métodos innovadores que permitan mejorar el aprendizaje y la experiencia tanto de los estudiantes como de los docentes en el actual método educativo virtual. 


\section{Listado de Referencia Bibliográfica}

Abreu, J. (2020). Tiempos de Coronavirus: La Educación en Línea como Respuesta a la Crisis (Times of Coronavirus: Online Education in Response to the Crisis). Daena: International Journal of Good Conscience, 15(1), 1-15. Recuperado de: http://www.spentamexico.org/ v15-n1/A1.15(1)1-15.pdf.

Alba-Linero, C., Moral-Sánchez, S. N., \& Gutierrez-Castillo, P. (2020). Impact of COVID-19 on education in a Spanish university: What should we change? In I. Sahin \& M. Shelley (Eds.), Educational practices during the COVID-19 viral outbreak: International perspectives (pp. 81-106). ISTES Organization.

Bao, W. (2020). COVID 19 and online teaching in higher education: A case study of Peking University. Human Behavior and Emerging Technologies, 2(2), 113-115. Disponible en: https://doi.org/10.1002/hbe2.191

González Velázquez, L. (2020). Estrés académico en estudiantes universitarios asociado a la pandemia por COVID-19. Revista Universidad Autónoma de Chiapas, Disponible en: https://espacioimasd.unach.mx/index.php/Inicio/article/view/249/782

Huang, Y., \& Zhao, N. (2020). Generalized anxiety disorder, depressive symptoms and sleep quality during COVID-19 outbreak in China: a web-based cross-sectional survey. Psychiatry Research, 20, 1-20. Morales-Holguín, A., y González-Bello, E. (2021). Diversidad de la enseñanza universitaria y de la práctica del diseño en México. Revista Actualidades Investigativas en Educación, 21(2), 1-23. doi. 10.15517/aie.v21i2.46779

Organización de las Naciones Unidas. (2020). La COVID-19 interrumpe la educación de más del 70\% de los jóvenes. Recuperado de: https://coronavirus.onu.org.mx/la-covid-19-interrumpe-la-educacion-de-mas-del-70-de-los-jovenes\#: :text=(COVID\%2D19)-,La\%20 COVID\%2D19\%20interrumpe\%20la\%20educaci\%C3\%B3n\%20de,del\%2070\%25\%20 de $\% 20 l o s \% 20$ j\%C3\%B3venes\&text=Los\%20efectos\%20desproporcionados\%20de\%20 la,la\%20Organizaci\%C3\%B3n\%20Internacional\%20del\%20Trabajo

Tovey, M. (2015). Developments in Design Pedagogy. International Conference on Engineering and Product Design Education, September 2015, United Kingdom: University Of Loughborough.

Unger, S., \& Meiran, W. R. (2020). Student attitudes towards online education during the COVID-19 viral outbreak of 2020: Distance learning in a time of social distance. International Journal of Technology in Education and Science (IJTES), 4(4), 256-266.

Uskun, E. Kisioglu, A. N., \& Ozturk, M. (2008). Stress and its effects on depression and anxiety among undergraduates. Primary Care and Community Psychiatry, 13(2), 73-82.

Valle Vargas, M. (2020). Habilidades emocionales necesarias en alumnos y profesores. En Tecreview Revista digitla. Recuperado de: https://tecreview.tec.mx/2020/06/15/tendencias/unam-60-de-alumnos-han-presentado-problemas-para-seguir-sus-clases-online/

Xhelili, P., Ibrahimi, E., Rruci, E., \& Sheme, K. (2021). Adaptation and perception of online learning during COVID-19 pandemic by Albanian university students. International Journal on Studies in Education (IJonSE), 3(2), 103-111. 
Zamora Saenz, I. B. (2020). Accesibilidad y uso de internet en México. La ENDUTIH a la luz de Covid-19. Visor Ciudadano 70. Recuperado de: http://bibliodigitalibd.senado.gob. $\mathrm{mx} /$ bitstream/handle/123456789/4869/VC_70.pdf? sequence $=1 \&$ isAllowed $=\mathrm{y}$

\begin{abstract}
The objective of this work is to know how the Design student adapted to virtual classes and what was his perception of his learning from the new reality product of COVID-19. More than half of the students consider that from a comprehensive perspective, online classes do not allow to achieve learning as in the face-to-face space, where the heterogeneity of the subjects and teaching typical of the design area is identified. It is suggested to implement innovative methods that allow improving the learning and experience of both students and teachers in the current virtual educational method.
\end{abstract}

Keywords: design - learning - virtual classes - higher education - university - COVID-19 - pandemic.

Resumo: O objetivo deste trabalho é conocer como o estudiante de Diseño se adaptar a las classes virtuais e cuál fue sua percepção de sua aprendizaje a partir de la nueva realidad producto del COVID-19. Más de la mitad de los estudiantes considera que desde uma perspectiva integral, las clases en linea não permiten alcanzar un aprendizaje como en el espacio presencial, donde se identifica la heterogeneidad de las asignaturas y enseñanza propia del area de diseño. Se sugerir implementar métodos inovadores que permitem melhorar o aprendizado e a experiência tanto de los estudiantes como de los docentes no método educativo virtual real.

Palavras chave: design - aprendizagem - aulas virtuais - ensino superior - universidade - COVID-19 - pandemia.

[Las traducciones de los abstracts fueron supervisadas por el autor de cada artículo] 